in type 2 diabetes provides a strong link between the high fat phenotype, and reduced energetic capacity of type 2 diabetic mitochondria.

\section{P33 PHARMACOLOGICAL STIMULATION OF HIF SIGNALLING AS A POTENTIAL TREATMENT APPROACH FOR THE TYPE 2 DIABETIC HEART}

MdL Sousa Fialho, M Kerr, MS Dodd, C Montes Aparicio, DJ Tyler, LC Heather. Department of Physiology, Anatomy and Genetics, University of Oxford

\subsection{6/heartjnl-2018-BSCR.38}

Introduction In response to ischemia, type 2 diabetic hearts are less able to upregulate anaerobic glycolysis and show poorer contractile function. Pharmacological activation of Hypoxia-Inducible Factor (HIF) may be beneficial for the diabetic heart, as it may promote glycolysis, improve function and tolerance of ischaemia.

Methods Control and type 2 diabetic rats were given five oral doses $(5 \mathrm{mg} / \mathrm{kg}$ daily) of PHD-selective HIF activator BAY853934. Hearts were perfused ex vivo and subjected to low-flow $(0.35 \mathrm{ml} / \mathrm{min} / \mathrm{gww})$ ischaemia-reperfusion (IR). Glycolysis, palmitate oxidation and function were measured throughout. Mitochondrial oxidation was measured in isolated mitochondria using a Clark-type electrode.

Results Diabetic hearts showed decreased rate-pressure product following ischaemia-reperfusion. Pre-treatment with BAY853934 resulted in increased glycolytic rate in diabetic hearts, paired with improved functional recovery post-IR. BAY853934 treated diabetic rats showed increased hematocrit, indicating systemic activation of HIF signalling. Blood glucose levels remained unchanged with treatment despite changes in cardiac glycolytic rate.

Conclusion This study has shown that treatment with HIF activators may provide a novel avenue to improve metabolism and functional recovery in the diabetic heart following ischaemia-reperfusion.

\section{P34 ADENOSINE A1 RECEPTOR ACTIVATION CAN PROTECT THE MYOCARDIUM FROM ISCHAEMIA REPERFUSION INJURY POST REPERFUSION}

J Bhandal, A Hussain, J Buckley, H Maddock. ABES, Coventry University

\subsection{6/heartjnl-2018-BSCR.39}

Introduction/background A1 adenosine receptors (A1AR), have previously been shown to limit injurious infarct development. The purpose of this current study was to determine if delayed activation of A1ARs from 0,15 or $30 \mathrm{~min}$ post-reperfusion can still limit infarct development.

Method Male Sprague-Dawley rats $(300 \pm 50 \mathrm{~g})$ were mounted on the Langendorff system. A1AR agonist, 2-chloro-N cyclopentyl-2'-methyladenosine (2'-MeCCPA) $\quad(0.1 \mathrm{nM}-1 \quad \mathrm{M})$ was administered at the onset of reperfusion as well as 15 and $30 \mathrm{~min}$ post-reperfusion $(10 \mathrm{nM})$ in the presence and absence of the A1AR antagonist 8-cyclopentyl-1,3-dipropylxanthine (DPCPX, $200 \mathrm{nM}$ ) as well as in the presence and absence of Wortmannin $(100 \mathrm{nM})$.

Results Administration of 2'-MeCCPA (1 nM-1 M) at reperfusion significantly reduced infarct size to risk ratio.
Furthermore, delaying the activation of A1AR to 15 or $30 \mathrm{~min}$ post-reperfusion significantly reduced infarct size compared to IR control.

The co-administration of DPCPX $(200 \mathrm{nM})$ with 2'MeCCPA $(10 \mathrm{nM})$ at the onset of reperfusion significantly attenuated the 2'-MeCCPA mediated infarct. The concomitant administration of 2'-MeCCPA with DPCPX at either 15 or $30 \mathrm{~min}$ post-reperfusion also abrogated 2'-MeCCPA induced cardioprotection.

Co-administration of Wortmannin (100 nM) with 2'MeCCPA $(10 \mathrm{nM})$ at the onset of reperfusion also significantly reduced the 2'-MeCCPA mediated infarct size as well as the co-administration at 15 or $30 \mathrm{~min}$ post-reperfusion reversed the cardioprotection.

Conclusion This is the first study to display how 2'-MeCCPA, a highly selective A1AR agonist, when administered at reperfusion, 15 or 30 min post-reperfusion can limit the infarct size development and how the RISK cell signalling pathway is also associated with cardioprotection.

\section{P35 TREATMENT WITH OLEIC ACID AND A PPAR $\alpha$ AGONIST INCREASES FATTY ACID OXIDATION IN BEATING HUMAN INDUCIBLE PLURIPOTENT STEM CELL-DERIVED CARDIOMYOCYTES}

H Al-Siddiqi, CA Lopez, LC Heather, CA Carr. Department of Physiology, Anatomy and Genetics, University of Oxford

\subsection{6/heartjnl-2018-BSCR.40}

Aim Human inducible pluripotent stem cell-derived cardiomyocytes (hiPSC-CM) have an immature phenotype including a glycolytic metabolism. Here we aimed to mature the metabolic status of beating (hiPSC-CM) by targeting the PPAR- $\alpha$ pathway, which regulates the transcription of genes involved in fatty acid metabolism. We cultured hiPSC-CM with the fatty acid Oleic Acid (OA) with or without the PPAR- $\alpha$ agonist WY14643.

Methods hiPSCs cells were differentiated into beating hiPSCCM by treatment with CHIR99021 followed by IWP4. Beating hiPSC-CM were treated with $400 \mathrm{uM}$ OA with or without $120 \mathrm{uM}$ WY14643 for 8 and 24 hours. Oxygen consumption was measured using a Clark-type oxygen electrode, glycolysis was measured by addition of $3 \mathrm{H}$-glucose. Gene expression was measured using qPCR.

Results A significant increase in oxygen consumption and reserve respiratory capacity when respiring on fatty acid was observed in beating iPSC-CM after culture with OA \pm WY14643 for 24 hours compared with untreated and OA-treated hiPSC-CM. OA \pm WY14643 caused a significant increase in expression of carnitine palmitoyltransferase $1 \mathrm{~B}$, medium chain acyl-CoA dehydrogenase and pyruvate dehydrogenase kinase after 8 hours whilst expression of glucose transporters and phosphofructokinase decreased. Addition of OA, but not OA \pm WY14643, increased the rate of O2 respiration on pyruvate and malate but not the reserve respiratory capacity. There was no change in the rate of glycoysis after treatment with OA \pm WY14643 for 24 hours.

Conclusion Treatment with OA and WY14643 for 24 hours increased fatty acid utilisation in beating hiPSC-derived cardiomyoyctes. Funded by the BHF and the Qatar Foundation. 